\title{
La gouvernance locale, un retour à la proximité
}

\section{Mme Nathalie Bertrand, M. Patrick Moquay}

\begin{abstract}
Local governance analysed through proximity - Economic and political analysis allows to study the theme of governance in regards of development dynamics and types of actors coordination involved. It questions directly the local dimension of the process. According to its theoretical background and operational implementation, governance is analysed through the notion of proximity, identifying problems and limits of its contribution to development objectives.
\end{abstract}

\section{Résumé}

Le rapprochement des analyses économique et politique permet de situer le thème de la gouvernance au croisement des dynamiques de développement et des formes de coordination entre acteurs, en soulignant la dimension locale du processus. A partir de ses fondements théoriques et de leur traduction opérationnelle, la gouvernance sera analysée à travers la notion de proximité, situant les problèmes et les limites de sa mobilisation au profit d'objectifs de développement.

\section{Citer ce document / Cite this document :}

Bertrand Nathalie, Moquay Patrick. La gouvernance locale, un retour à la proximité. In: Économie rurale. N²80, 2004. Proximité et territoires. pp. 77-95;

doi : https://doi.org/10.3406/ecoru.2004.5474

https://www.persee.fr/doc/ecoru_0013-0559_2004_num_280_1_5474

Fichier pdf généré le 09/05/2018 


\section{a gouvernance locale, un retour à la proximité}

Nathalie BERTRAND • Cemagref, Développement des territoires montagnards, Grenoble Patrick MOQUAY - École nationale du génie rural des eaux et des forêts, Engref, Clermont-Ferrand

Le thème de la « gouvernance » a fait l'objet de nombreux débats et la notion est intrinsèquement polysémique. Elle interpelle tout particulièrement les sciences économique et politique, tant en terme disciplinaire que sur le plan des champs théoriques investis.

Ainsi en science économique, la gouvernance a connu certaines prémices et se trouve évoquée - pas toujours clairement comme le souligne Wallet (1997) - dans deux grandes approches théoriques des dynamiques des espaces économiques et des mécanismes de coordination : la théorie des contrats (Williamson, 1981) et la théorie de la régulation. Dans la première, la gouvernance est définie comme une structure contractuelle permettant aux acteurs de se coordonner dans le cadre de relations économiques ; dans la seconde, la gouvernance apparaît comme une structure politique, représentée essentiellement par l'État, intervenant localement dans la réalisation du développement économique local. Les régulationistes n'opposent pas institutions et marché mais analysent comment la présence d'institutions non économiques peut faciliter la coordination des agents (Villeval, 1995).

En science politique, le terme de gouvernance a d'abord été utilisé pour désigner les formes de régulation de systèmes complexes ou fragmentés, notamment à l'ćchcllc mćtropolitaine ou en matière de relations internationales. À la suite de la mise en évidence empirique du caractère composite des systèmes d'acteurs en cause, la gouvernance recouvre tout système de décision publique intégrant des acteurs privés. Par extension, un usage normatif du terme s'est développé, et se résume à l'appel à un État modeste. Cet usage normatif a lui-même deux facettes : une version managériale qui préconise un État aux pouvoirs diminués, centré sur l'accompagnement des acteurs privés ; une version démocratique qui insiste, pour sa part, sur la nécessaire expression des acteurs concernés et leur participation à la définition et à la mise en œuvre des actions publiques, sans que cela exige en soi une réduction des attributions des pouvoirs publics.

Finalement, la diversité des emplois du terme est grande. On peut même considérer, comme Lorrain (1998), qu'il y a contradiction entre les usages : en sciences économiques, la gouvernance vise avant tout à expliquer des formes d'organisation complémentaires au fonctionnement du marché, voire parfois plus rigides que celui-ci ; en sciences politiques, la gouvernance renvoie au contraire à des formes de coordination complémentaires à l'intervention étatique, mais plus souples que les institutions publiques. L'inspiration semble donc contraire, mais peut tout autant être interprétée comme un rapprochement, par mutuelle prise en compte, des analyses économiques et politiques.

La gouvernance se trouve ainsi à la confluence de forces économiques - puisqu'elle est relative soit au fait productif luimême, soit plus globalement aux dyna- 
miques de développement - et de forces sociales et politiques - puisqu'il s'agit de pointer et d'analyser des formes de relations de coordination entre divers acteurs, visant précisément à orienter ces dynamiques de développement. D'une manière générale, on peut la caractériser en suivant Benko et Lipietz (1992): «Il s'agit de toutes les formes de régulation qui ne sont ni marchandes, ni étatiques. Pour paraphraser la définition de Gramsci (l'État = société civile + société politique), la gouvernance, c'est la société civile moins le marché... plus, il faut bien l'ajouter, la société politique locale, les notables, les municipalités! »Comme forme de régulation ni marchande ni étatique, elle apparaît comme le résultat dynamique de tensions entre des régularités verticales de type sectoriel et macro-économique, et des régularités horizontales caractéristiques des dynamiques endogènes des espaces économiques locaux (Gilly et Pecqueur, 1997).

Cette construction, à la fois globale et locale, pose alors très directement la question de ces dynamiques endogènes. Elle met d'emblée l'accent sur la dimension localisée de la gouvernance, qui replace les phénomènes économiques dans leur inscription à la fois sociale (Bagnasco et Trigilia, 1993) et spatiale. La gouvernance recoupe en cela certains approfondissements de l'analyse économique des dernières décennies, qui se sont traduits par la prise en compte de plus en plus complexe de la dimension spatiale. L'analyse est ainsi passée de l'espace caractérisé par le coût de distance au concept de territoire, acteur du développement économique. Le territoire, porteur de construits socio-économiques et institutionnels ancrés spatialement, constitue aujourd'hui un niveau de régulation infranational (Corolleur et Pecqueur, 1996). Le constat de la diversité des configurations organisationnelles et l'analyse des dynamiques économiques locales ont ainsi mis sur le devant de la scène les mécanismes de coordination qui se construisent entre acteurs de natures diverses.
La question est alors posée : quelle est la dimension politique de la dynamique des espaces économiques locaux (Soulage, 1994 ; Berthet et Palard, 1997) et quel est le rôle des acteurs publics?

Dans cet article, nous écarterons les acceptions de la gouvernance qui ne s'intéressent qu'aux modalités de relations interentreprises ou intra-entreprises (dans les sciences de gestion), pour nous attacher aux différentes formes de coordination entre acteurs de statut divers, visant à piloter ou à orienter le développement des territoires. Ce constat de formes différenciées d'articulation entre acteurs renvoie à la question du développement local. Le thème de la gouvernance locale y représente une recherche de nouveaux modes d'organisation territoriale et une conception moderne du management local, transcendant les politiques sectorielles. De plus, les changements notables qui ont affecté, ces dernières années, les conceptions de l'action publique, sont au cœur d'un déplacement de l'analyse de la seule action des gouvernements (ou, plus largement, des institutions publiques) vers des formes de mobilisation d'acteurs privés (associations, entreprises, simples citoyens) contribuant collectivement à la régulation des espaces et des activités.

Ainsi, au-delà de la diversité de cette action publique, la gouvernance locale recouvre des mécanismes de coordination et d'action collective qui permettent de mobiliser un ou des champs d'interaction entre des acteurs de nature variée et de garantir la tenue d'accords, sur un projet plus ou moins explicite, entre partenaires destinés à être partie prenante du gouvernement local. Dans cette perspective, l'émergence du thème de la gouvernance locale peut être lue comme « un retour de la proximité », aussi bien dans les analyses théoriques que dans les politiques ou pratiques qui les déclinent.

La notion de proximité n'est pas univoque ; elle renvoie à la fois à la proximité organisationnelle - obéissant à des logiques de similitude ou d'appartenance - et à la 
proximité géographique - séparation dans l'espace des liens évalués en termes de distance physique ou temporelle -. C'est le lien entre développement local, gouvernance locale et proximités qui va nous intéresser ici.

Pour cela et dans un premier temps, nous nous interrogerons sur les fondements théoriques de la gouvernance locale, qui peut permettre un rapprochement entre approches disciplinaires relevant des sciences économiques et des sciences politiques (I). Nous montrerons ensuite que la mobilisation du concept est liée à la façon de penser et de mettre en scène les proximités autour (ou au sein) de l'action collective (II). Puis nous nous intéresserons à la traduction opérationnelle du concept au regard d'un certain nombre de dispositifs liés aux politiques de développement local, des procédures contractuelles aux réseaux et aux dispositifs de démocratie participative (III). À la lumière de l'exemple de ces dispositifs pratiques et en conclusion, la question des limites du rapprochement entre proximités et gouvernance sera discutée

\section{Convergence vers la notion de gouvernance locale}

D'un point de vue analytique, le terme « gouvernance » conduit à s'interroger, dans un contexte organisé des échanges, sur les modes de coordination entre acteurs et institutions, et les formes de développement territorial qui en résultent (Offner, 1999). Il s'insère donc dans les approches disciplinaires qui traitent de ces modes de coordination et de ces formes de développement.

L'analyse s'attache notamment aux formes de mobilisation d'acteurs privés (associations, entreprises, simples citoyens) cherchant à contribuer à la régulation collective des espaces et des activités. Le concept de « gouvernance locale » permet de renouveler la réflexion sur le gouvernement des territoires, leurs modes de gestion et d'administration. Il pose la question de l'au- tonomie relative des processus de développement locaux et de la nature ou du statut des acteurs concourant à l'orientation du développement. Ces interrogations émergent aussi bien dans le domaine des sciences économiques que dans celui des sciences politiques.

\section{La gouvernance locale comme facteur de compréhension des mécanismes économiques}

La gouvernance locale, notion mobilisée assez tardivement en économie, est porteuse de deux débats. Le premier est celui du fait productif et de la nature des coordinations entre agents individuels ou collectifs (Coase, 1937 ; Commons, 1934 ; Williamson, 1975), dès lors qu'est rejetée une position standard considérant le seul prix du marché dans les échanges. Le second est celui de la prise en compte de la dimension spatiale comme facteur intrinsèque du fait productif. Ce dernier aspect, central pour repenser les phénomènes macro-économiques à partir d'un ancrage local, n'est cependant pas nouveau'.

Le problème de la localisation des unités de production, thème traditionnel de l'économie spatiale, envisage tout d'abord l'espace comme un élément « passif », analytique, qui entre dans le calcul économique des agents. Il constitue un coût au regard de

1. Dockès (1969) souligne que la dimension spatiale est déjà présente dans les analyses classiques des $\mathrm{XVI}^{\mathrm{c}}$ et XVIII' siècles et se rapproche de certaines recherches contemporaines (équilibre et déséquilibre spatial ; intégration des déplacements des hommes, des capitaux et des entreprises; importance du rôle de l'État dans le maintien de ces déséquilibres). Elle a cependant longtemps occupé une place aux marges de l'économie (Thisse, 1996) et la participation d'une science de l'espace économique à l'explication économique d'ensemble est relativement récente. Selon les perspectives d'analyse, la façon d'introduire la dimension spatiale va, au cours du $\mathrm{XX}^{\mathrm{e}}$ siècle, vers une complexification croissante des rapports de l'analyse économique au spatial, tant pour les économistes industriels que pour les économistes régionaux. 
ses caractéristiques propres (une distance ou des propriétés économiques affectant de manière exogène l'espace et le différenciant). Cette perspective emprunte aux approches néo-classiques deux entrées : pour l'une, introduite historiquement par Von Thünen, l'espace est une surface topologique, consommable, orientée par un ou plusieurs centres ; pour l'autre, l'espace est considéré comme un ensemble de lieux séparés par la distance, ayant des propriétés économiques particulières (disponibilité de facteurs de production ou existence de marchés finaux) et l'analyse est centrée sur la minimisation des coûts de transport -analyse issue des travaux de Weber (1929). Cette perspective relève également $d$ 'approches hétérodoxes à travers la théorie de la division spatiale du travail (Aydalot, 1984).

Située au croisement de l'économie industrielle et de l'économie spatiale, la dimension spatiale devient un élément constitutif des mécanismes économiques. - Les analyses relevant de la compétitivité entre pays - voire entre régions face à la mondialisation des échanges - intègrent l'élément spatial comme une caractéristique intrinsèque des processus économiques. L'espace devient un élément à part entière des modes d'organisations industrielles. Il constitue un acteur économique particulier, le « territoire ». Les modèles de concurrence territoriale, avant tout conçus comme une instance de décision publique, s'enracinent dans l'économie publique locale, croisant les stratégies de localisation des firmes et des problèmes d'organisation industrielle (Rallet et Torre, 1995).

- Les analyses en termes de concurrence territoriale appréhendent les actions destinées à attirer des activités ou à mobiliser des ressources locales. Ces approches peuvent être classées en trois ordres selon les problèmes complexes traités (Jayet, 1994) : - les caractéristiques de l'offre territoriale de site (offre de terrains, offre de biens publics locaux, offre de « biens organisationnels »);
- les processus qui matérialisent les stratégies de localisation en réponse à cette offre de site,

- l'attractivité du site liée à l'organisation territoriale entre institutions locales et les autres agents au travers de processus de coopération.

De plus, l'économie géographique (Krugman, 1991 ; Thisse, 1996) ouvre un nouveau champ de réflexion sur la dimension spatiale (Rallet, 2000), remettant en cause le postulat de concurrence parfaite et la reconnaissance de phénomènes d'agglomération et de rendements croissants. L'existence d'externalités, créant des rendements croissants à l'échelle d'un territoire ou sur la différenciation des produits et des services, génère des processus inégaux d'agglomération, et constitue des " substituts concurrentiels » à la décroissance des coûts de transports (Rallet et Torre, 1995).

Enfin, l'approche territoriale propose de fonder le territoire sur un mode particulier d'organisation industrielle à travers deux courants, celui des districts industriels (Marshall, 1920) revisités dans les années 197080 par des économistes italiens (dont Becattini, 1989), américains (Piore et Sabel, 1984) et français (Courlet et Pecqueur, 1989) et celui des milieux innovateurs (programmes de recherche Gremi I, II, III). L'hypothèse centrale repose sur le fait que le territoire ait la capacité d'endogénéiser son développement au moyen des modes coopératifs de petites et moyennes entreprises fondées sur des interdépendances entre agents et entre agents et institutions. Les analyses sur l'organisation économique de l'espace relancent ainsi, pour les économistes industriels et les économistes régionaux, le concept de gouvernance locale comme forme de régulation territoriale et d'interdépendance dynamique entre agents productifs et institutions locales. L'espace devient pour les premiers une dimension à part entière du fait productif ; pour les seconds, c'est la dimension productive de l'espace qui est mise en avant. 


\section{La gouvernance locale comme modalité de gouvernement}

En science politique, la conceptualisation de la gouvernance territoriale est issue de l'analyse du gouvernement local et, plus spécialement, du gouvernement urbain. Le recours à la notion de gouvernance entend avant tout caractériser les activités de gouvernement (ou de régulation) de systèmes complexes ou fragmentés ${ }^{2}$.

Le terme apparaît pour analyser notamment les modalités de gouvernement des ensembles métropolitains (Lefèvre, 1998 ; Jouve, 1999). Dans cet usage, le terme de gouvernance ne se différencie pas toujours de la notion de gouvernement, car l'accent reste porté sur les arrangements institutionnels (et en l'occurrence inter-institutionnels) à l'échelle des grandes agglomérations (Parks et Oakerson, 1989 ; Dente, 1990). Cette équivalence des termes gouvernance et gouvernement sont fréquente dans les travaux américains, pour des raisons de tradition intellectuelle sur lesquelles nous reviendrons.

Mais au-delà des situations de fragmentation institutionnelle caractéristiques de la plupart des agglomérations, la notion de gouvernance est également mobilisée pour rendre compte de la pluralité des acteurs associés à la définition de l'action publique, en premier lieu celle du milieu urbain. L'étude des modalités de détermination des logiques de développement urbain a mis en

2. Certains auteurs, notamment Padioleau (1999, 2000) associent principalement la gouvernance à une conception utilitariste de l'action publique, liée à l'affirmation d'un idéal de performance. Cela le conduit à dénoncer la gouvernance comme un affaiblissement du politique au profit d'une gestion à caractère technocratique. Cette conception émergente se rattache plutôt, selon nous, à la diffusion des thèses du « nouveau management public ", inspiré des pratiques des entreprises (Cannac et Godet, 2001). Ces nouveaux modèles de management public apparaissent concurremment aux changements auxquels nous nous intéressons, mais ne s'y ramènent pas. évidence les liens unissant décideurs publics et acteurs privés, déterminant des formes stables de relations que l'on a pu qualifier de régimes urbains (Stone, 1989) ou de coalitions de croissance ${ }^{3}$ (Logan et Molotch, 1987). Stone (1993) souligne que l'élection ne suffit pas à donner aux décideurs publics la capacité d'agir ; cette capacité doit être construite dans une relation avec l'ensemble (ou du moins une partie) des acteurs susceptibles de contribuer à la mise en œuvre des actions envisagées. Dans le prolongement de ces travaux américains, l'usage de la notion de gouvernance en Europe entend souligner la participation de divers intérêts privés aux systèmes de décision publics régissant les agglomérations et, par extension, l'action publique territoriale (Padioleau, 1991).

Ces approches nouvelles du gouvernement territorial et des processus productifs, articulant acteurs institutionnels publics et acteurs privés, rapprochent les perspectives disciplinaires et peuvent se retrouver autour de la notion de gouvernance locale. Bien que fondamentalement inscrite dans la structuration locale des phénomènes productifs, celle-ci peut également rendre compte de l'articulation entre les relations locales et leur contexte global.

\section{Les tensions entre proximité géographique et contexte global au centre de la gouvernance locale}

La notion de gouvernance locale introduit la question de l'autonomie relative des pro-

3. Les coalitions de croissance (growth coalitions) désignent les coalitions d'acteurs favorables à la croissance urbaine, dans sa double dimension économique et démographique. Ici encore, il convient de dissocier le mécanisme (la coalition) et l'affectation de ses finalités (la croissance). L'observation empirique a en effet montré l'existence d'accords similaires entre des acteurs sociaux choisissant délibérément de limiter la croissance, voire de s'y opposer (anti-growth coalitions), par exemple pour éviter l'arrivée de nouvelles populations ou les perturbations du marché foncier. 
cessus locaux de développement. Cette autonomie relative s'oppose aux théories holistiques qui voient les territoires comme totalement dépendants de processus de globalisation. Elle suppose de dépasser les approches localistes qui voient les régularités macro-économiques comme résultant seulement de la juxtaposition de formes locales de régulation, sans envisager une structuration mutuelle des deux niveaux. La gouvernance locale, dans son contenu programmatique et ses effets opérationnels, est le résultat dynamique d'une construction à la fois globale et locale, d'acteurs clefs qui jouent sur l'espace économique local : établissements appartenant à de grands groupes, dévolus aux services publics, aux syndicats... (Gilly et Wallet, 2001).

La conception de la gouvernance locale permet d'intégrer mécanismes productifs et mécanismes institutionnels à la fois dans la dimension locale liant proximité géographique - faisant référence à l'espace géonomique de Perroux (1955) et à la distance fonctionnelle - et proximité organisationnelle - reposant sur une logique de similitude et une logique d'appartenance (Gilly et Torre, 2000), dans la dimension local-global (proximité organisée entre acteurs locaux et acteurs extérieurs au territoire). Cette notion de gouvernance locale n'est pas un processus seulement endogène, par l'incorporation des rapports entre institutions locales (formelles et informelles) et formes institutionnelles globales. Le rôle décisif joué par les institutions formelles, notamment les collectivités locales, est souligné, orientant "les comportements des agents économiques et...[contribuant]... à faire émerger ou perdurer la gouvernance territoriale" (Gilly et Torre, 2000). L'analyse en termes de gouvernance locale rapproche sciences économiques et politiques : objets partagés, prise en compte mutuelle des perspectives, attention commune aux dimensions de proximité.

\section{La gouvernance locale : une construction de l'action collective par un retour aux proximités}

La gouvernance locale, explicitement pour l'économie ou implicitement pour les approches politiques, repose sur l'activation, et donc la mise en évidence, de relations de proximité. Cette attention à la proximité, formalisée en particulier au sein du groupe des « Dynamiques de proximité »", répond à un besoin de théoriser le territoire. Elle pcrmet de resituer la gouvernance dans un nouveau contexte d'analyse et de définition. À partir notamment du débat sur la nature polysémique de la proximité - intégrant des dimensions sociale et économique, ces travaux posent la question des formes de relations entre acteurs et de l'activation de ces relations, permettant d'aborder de façon renouvelée les questions de coordination (Gilly et Torre, 2000).

\section{Le caractère composite du système local d'action publique}

L'action publique locale n'est plus présentée comme la seule responsabilité de l'institution municipale (ou départementale), mais comme la résultante d'un processus dc coopération ou de coordination entre de nombreux acteurs et opérateurs (Gaudin et Novarina, 1997). Si la collectivité locale garde un rôle d'orientation et de pilotage, elle compose avec d'autres institutions, publiques ou privées, obéissant à leurs propres logiques d'intérêt et/ou exerçant des responsabilités sur des domaines de compétences tantôt partagés, tantôt disputés, mais jamais absolument étanches ni autonomes. Gilly et Leroux (1999) parlent ainsi de compromis institutionnels composites qui orientent la coordination des acteurs selon des « régularités technico-écono-

4. Parmi les références majeures du groupe, $c f$. Gilly et Torre (2000) ; le numéro spécial de la RERU (1993), le colloque «Proximité et coordination économique », Lyon, 5-6 mai 1997. 
miques et sociales géographiquement concentrées ».

Dans le cas de la construction de politiques locales de reconversion industrielle, par exemple, l'analyse montre que le processus de mise en œuvre de l'action publique prend la forme d'une construction sociale complexe, et sort d'un schéma linéaire : diagnostic de crise - projets de reconversion - action (Grossetti et Beslay, 1999). L'action publique intègre des éléments de diverses natures tels que des relations entre organisations publiques ou privées, des réseaux inter-individuels, des « références discursives collectives » (ou mythes), renforçant l'idée d'une imbrication des dimensions aussi bien politiques et économiques que sociales.

En sciences économiques, l'analyse des mécanismes de coordination, au regard du concept de gouvernance locale, souligne également la complexité du phénomène apprécié à la fois à l'articulation de coordinations économiques et politiques, et dans une dialectique locale-globale. Les territoires de développement inscrits aux Contrats de plan en offrent un exemple. Sous l'égide du Conseil régional, ces contrats définissent des projets à l'échelle de territoires infra-régionaux. Ces contrats locaux créent des relations partenariales entre les acteurs du secteur privé et/ou les structures intercommunales, établissant alors des formes de coordinations " mixtes» entre acteurs économiques et acteurs politiques (Gilly et Wallet, 2001). D'autres procédures contractuelles peuvent être soulignées de façon analogue.

En sciences politiques, la notion de gouvernance locale met d'abord l'accent sur le caractère composite du système d'action présidant à l'élaboration des politiques locales d'aménagement et de développement. Les collectivités locales, si elles disposent en droit du pouvoir de définir ces orientations, prennent garde d'y associer les représentants des différents milieux concernés. Les politiques publiques locales, de ce fait, sont issues de processus de construction collective associant divers acteurs, porteurs d'intérêts différents ; et l'analyse des institutions locales doit, en conséquence, laisser place à l'analyse des systèmes locaux d'action au sein desquels s'élaborent les politiques (Mabileau, 1994 ; Beslay et al., 1998). L'attention portée aux processus collectifs d'action localisée souligne une différence intellectuelle entre les analyses françaises et américaines.

Aux États-Unis, l'accent s'est porté très tôt sur le rôle des groupes d'intérêt, des lobbies, dans les processus publics de décision. Dans l'analyse du gouvernement local notamment, les différents courants théoriques se rejoignent pour constater l'étroite imbrication entre décideurs publics et responsables privés. Les politiques locales y résultent de rapports de force entre groupes, où les acteurs économiques jouent un rôle de premier plan (Polsby, 1980). Le concept de gouvernance est plutôt en continuité des principaux courants d'analyse, dont il ne fait qu'exprimer une préoccupation générique, celle de la constitution d'un système intégré mais composite de règlement des affaires locales. Dans cette perspective, la spécificité des institutions publiques disparaît, au point que l'analyse de l'effet propre des institutions a pu passer, dans les années 1980, sous l'appellation de néo-institutionnalisme (Scokpol, 1985), pour un réel renouveau conceptuel.

En France, le terme de gouvernement renvoie à une approche institutionnelle, tandis que la notion de gouvernance amène plutôt à se détacher des seules institutions locales, pour envisager le système décisionnel plus large qui préside à l'action locale (Le Galès, 1995). Là encore, mais sans renoncer à la place centrale qu'occupent les décideurs politiques (notamment urbains), on s'attache à rendre compte des processus de décision comme impliquant une pluralité d'acteurs et d'intérêts. 


\section{L'articulation entre institutions politiques locales et acteurs économiques}

Dans cet esprit, le concept de gouvernance locale met l'accent sur les liens étroits qui associent les décideurs publics et les représentants des différents autres acteurs, notamment les responsables d'entreprises. On doit relever de ce point de vue une nouvelle différence entre le débat français et le débat américain. La discussion porte notamment sur l'autonomie relative des collectivités locales et sur le poids des acteurs privés dans les processus urbains de décision (Le Galès, op. cit.).

D'un point de vue factuel, les budgets des villes américaines sont essentiellement alimentés par l'impôt foncier, et le produit de celui-ci est très dépendant de la situation économique de la collectivité. Dans une situation où la mobilité résidentielle des ménages comme des entreprises est relativement ouverte, la bonne santé des finances locales dépend de la satisfaction des contribuables, et les milieux économiques peuvent trouver dans ce mécanisme un levier pour faire prendre en compte leurs intérêts. L'autonomie des gouvernements locaux vis-à-vis des entrepreneurs privés serait donc structurellement faible aux Etats-Unis (Gurr et King, 1987 ; Peterson, 1981), ce qui justifie l'attention originelle portée aux phénomènes de gouvernance, entendus comme la participation d'une pluralité d'agents aux processus de décision.

A contrario, les collectivités françaises (ou plus largement européennes) sont dans une situation de moindre dépendance par rapport aux acteurs économiques (Wolman et Goldsmith, 1990). Cela tient à la relative diversité des sources de financement des collectivités et, notamment, à la place importante que continuent de tenir les financements étatiques dans les budgets locaux. Ainsi, la situation européenne se caractérise par des institutions municipales urbaines toujours puissantes, voire renforcées en France ces dernières décennies par la décentralisation (Lorrain, 1989, 1991). Les élus municipaux et les autres institutions publiques pourraient dès lors paraître suffisamment autonomes dans leurs choix pour que l'on fasse l'économie d'une prise en compte des relations qui les lient aux autres acteurs de l'agglomération. Pourtant, l'autonomie relative des responsables urbains ne doit pas masquer le rôle (parfois ancien, d'ailleurs) que jouent divers acteurs privés ou semi-publics dans la détermination des choix d'aménagement et des orientations de développement des collectivités.

Dans les agglomérations, on a ainsi vu l'implication croissante des opérateurs de réseaux, devenus de grandes entreprises de services, ou encore l'émergence d'une myriade de sociétés d'économie mixte associant les collectivités à quelques grands investisseurs (en particulier la Caisse des dépôts et consignations). Jouve (1999) souligne que le poids des acteurs économiques a souvent été déterminant dans la mise en place de nouvelles institutions d'agglomérations à travers l'Europe. Les acteurs économiques sont à la recherche d'interlocuteurs institutionnels susceptibles de stabiliser leur cadre d'activité et de soutenir leurs stratégies de développement. Dès lors, ils développent des relations avec les acteurs politiques métropolitains, en mobilisant les canaux d'expression dont ils disposent et en activant les divers réseaux qui peuvent les relier. Le rôle politique des Chambres de commerce et d'industrie a, par exemple, été souligné de longue date, comme l'ont illustré les cas de Dunkerque (Castells et Godard, 1974) ou de Bordeaux (Lagroye, 1971). Ce mouvement d'association entre décideurs publics et acteurs privés a eu tendance à se renforcer durant les dernières décennies.

Les travaux les plus récents d'analyse de l'action publique témoignent ainsi, au-delà des seules agglomérations, de l'ouverture des processus de décision et de la formalisation de ces relations de proximité entre acteurs publics et privés. L'appel aux partenariats (Ruegg, Decoutère et Mettan, 
1994), l'élaboration de projets territoriaux engageant des acteurs de statuts divers, la généralisation des procédures contractuelles (Gaudin, 1996) attestent de cette association des acteurs privés à l'élaboration, voire à la mise en œuvre, de l'action publique.

\section{Les modalités de l'action collective}

Ces analyses des processus locaux de décision renvoient à des formes d'action collective reposant sur des jeux de négociations, de compromis, d'alliances, qui supposent l'activation de relations et de réseaux divers. Ces mécanismes se rattachent tout d'abord à la proximité géographique des acteurs urbains (ou locaux, ou départementaux...). C'est en effet du fait de leur ancrage territorial que les acteurs se rencontrent, dans la mesure cependant où leurs intérêts respectifs peuvent relever de décisions locales, qui les amènent à s'engager dans des démarches locales de pilotage du développement ${ }^{6}$. La proximité géographique est donc un préalable, puisque

5. Relations contractuelles entre institutions publiques à l'origine, mais engageant de plus en plus souvent de grandes entreprises publiques et des représentants des divers acteurs locaux, par exemple sous la forme de chartes.

6. Les entreprises de grande taille ont un comportement ambivalent de ce point de vue : elles peuvent adopter une forme d'investissement dans la politique locale, pour des raisons à la fois économiques (s'assurer des conditions optimales d'exploitation) et socio-politiques (assumer une forme de responsabilité sur la société locale, qui peut constituer une charge mais également un atout dans le jeu politique local) ; mais elles peuvent également refuser cet ancrage territorial, pour ne se référer qu'au fonctionnement des marchés, et ne considérer que les conditions de la compétition entre leurs sites d'implantation potentiels. La construction par les acteurs économiques de relations de proximité organisée puis la participation active à des processus de gouvernance répond en fait à la perception stratégique qu'ils développent de leurs intérêts locaux et de leurs alternatives (Moquay et Palard, 1990). c'est elle qui définit la nature de l'intérêt à agir et fonde l'implication locale des acteurs.

Cette proximité géographique cependant ne joue ici que comme élément de contexte ou de cadrage : les mécanismes d'action collective s'appuient avant tout sur l'activation d'une proximité organisée, selon différentes logiques. Les échanges entre acteurs prennent place au sein de réseaux d'influence ou d'instances formalisées de concertation, qui relèvent d'une logique d'appartenance. Ces réseaux d'appartenance peuvent être expressément constitués en vue de contribuer au règlement des affaires locales (clubs d'entreprise, associations de développement...). Ils peuvent aussi se manifester à partir de structures a priori extérieures au champ d'action considéré (activités sportives ou culturelles, réseaux professionnels ou amicaux, etc.).

La simple appartenance à un territoire peut également être le ferment identitaire de l'engagement dans l'action (Moquay, 1998), auquel cas la mobilisation renvoie à la proximité organisée par le biais de la logique de similitude. Cette dernière suppose tout un travail conceptuel ou idéologique préalable, destiné à construire l'archétype de l'identité locale - et surtout de l'intérêt local - et à le diffuser au sein de certains groupes locaux.

Les projets collectifs peuvent ainsi être une source d'activation de la proximité géographique. Filippi et Torre (2003) montrent - à travers l'exemple de la mise en place des Réseaux de diffusion technologique (RDT) - que la seule proximité géographique est impuissante à générer l'interaction et les synergies locales au sein des tissus locaux, d'où l'échec de certaines politiques publiques de soutien à l'innovation. Les auteurs soulignent l'importance du projet commun, les « actions collectives qui mobilisent des compétences ou valorisent des savoir-faire d'origine locale » en générant des processus de coopération. 
La proximité organisationnelle souligne l'intérêt d'une prise en compte des relations rapprochant les divers acteurs locaux ${ }^{7}$, à travers des institutions formelles, des réseaux, des relations économiques ou des rapports d'influence et de dépendance. L'analyse des formes de gouvernance et de construction de l'action publique locale met en évidence les différents aspects de la dimension collective du projet : collective par les différentes institutions publiques y participant, collective par l'implication d'acteurs économiques, collective enfin en réintégrant dans le processus l'initiative individuelle. Ces trois facettes du projet collectif font appel aux dimensions organisationnelle et géographique des relations de proximité.

\section{Traductions opérationnelles et dispositifs mis en œuvre :} les échelles de la gouvernance locale et celles de la proximité

La gouvernance locale et la question de l'action collective interrogent cependant les échelles auxquelles se construit cette capacité d'organisation et ces modalités pratiques de mise en œuvre collective ${ }^{8}$. Ces échelles sont variées et les trois modalités pratiques présentées ici nous semblent refléter de manière concrète cette diversité. Elles caractérisent différents processus opérationnels dans lesquels s'engagent les acteurs locaux au nom de la gouvernance.

- La première modalité souligne la formalisation des coordinations par les procédures contractuelles. Ces contrats locaux

7. Rappelons que ces relations n'associent pas que des acteurs locaux, mais incluent des acteurs extérieurs au territoire, inscrivant celui-ci dans des relations local-global.

8. Cette partie est issue des réflexions menées en commun avec Yves Gorgeu (Mairie-conseils, Caisse des dépôts et consignations) dans le cadre du groupe de prospective « Espaces naturels et ruraux et société urbanisée » (programme de prospective de la DATAR Territoires 2020, 2000-2003). cristallisent des financements mais conduisent également à instaurer de nouveaux rapports entre acteurs.

- La deuxième s'intéresse aux coordinations résiliaires, à travers leurs formes locales et leurs modes de fonctionnement. La mise en œuvre de réseaux locaux est, en effet, encouragée par un certain nombre d'initiatives publiques.

- La troisième traite des dispositifs de démocratie participative, valorisés et renforcés ces dernières années par l'émergence de nouvelles politiques (politique de la ville, conseils de quartiers, pays et conseils de développement...).

\section{Les procédures contractuelles}

Des dispositifs novateurs ressortant de la gouvernance, en tant qu'elle recouvre les modalités de coordination entre acteurs au sein d'ensembles institutionnels complexes, se sont développés ces trente dernières années, avec une nette accélération plus récemment. Il s'agit des politiques contractuelles par lesquelles l'État ou d'autres institutions publiques planifient et formalisent leurs relations avec divers partenaires publics ou privés (Gaudin, 1996).

Avec le ralentissement de la croissance et la crise des années 1970 , les pouvoirs publics (notamment la DATAR) ont commencé à s'intéresser aux initiatives de développement local, en formulant de premières politiques contractuelles en direction des associations locales de développement regroupant élus et responsables socio-professionnels. Deux procédures pionnières proposées par l'État, les Plans d'aménagement rural (PAR) et les Contrats de pays, ont permis de structurer des accords publicsprivés locaux, plus ou moins ambitieux selon les territoires. Dans un jeu triangulaire inauguré au niveau régional avec l'appel aux « forces vives », l'État tentait d'équilibrer sa relation avec les élus locaux par l'association d'un tiers acteur représentant les activités économiques et sociales du territoire. La décentralisation et la régionali- 
sation (dès 1978) des politiques contractuelles territoriales, puis les interventions européennes, notamment avec le programme d'initiative communautaire Leader, ont suscité de multiples initiatives de gouvernance mixte (public-privé) pour gérer des projets et des contrats.

Ces initiatives ont depuis connu une forme de normalisation institutionnelle, se transformant en une simple procédure administrative, bien plus qu'un accord de gouvernance fixant des orientations de développement partagées. Les politiques contractuelles sont devenues des moyens classiques pour accéder aux financements, au détriment de la qualité des démarches privilégiant dialogue, collaboration et innovation. De plus, les régions et l'État ont progressivement exigé l'abandon des formules associatives au profit d'établissements publics, offrant plus de garanties pour gérer les fonds publics. Ces évolutions se sont accompagnées d'un recul important quant à la place des citoyens et du monde associatif dans les projets et les actions portées par ces procédures contractuelles. Avec le développement de l'intercommunalité à fiscalité propre depuis 1992, les communautés de communes portent de plus en plus directement les actions de développement, en oubliant le dialogue avec les acteurs de la société civile ou en les entraînant dans des logiques d'instrumentalisation qui les transforment trop souvent en de simples auxiliaires de leur politique.

Le principe contractuel n'en a pas moins été réaffirmé et sollicité de manière croissante. La décentralisation, en accroissant l'autonomie des différents niveaux de collectivités, a renforcé les besoins de coordination et fait de la démarche contractuelle une solution privilégiée. De là sont nés, par exemple, les Contrats de plan État-région (CPER). La mise en place des nouveaux contrats de pays, contrats d'agglomération et contrats de ville, déclinaisons territoriales des CPER, vient conforter l'édifice contractuel. De plus, l'État a également initié une politique de contractualisation avec les grands établissements publics ou les entreprises publiques, de façon à se donner un cadre de moyen terme régissant les relations entre institutions et encadrant les choix d'intérêt mutuel. Ces formes de contractualisation institutionnalisées s'articulent essentiellement autour du niveau régional, signataire, d'une part avec l'État central et, d'autre part, avec des échelons infra-régionaux. On observe ainsi une forme de décalque territorial, décentralisé, d'une contractualisation régionale censée organiser et planifier les stratégies d'aménagement et de développement.

Ce déploiement des politiques contractuelles passe enfin par l'apparition de formes multiples de chartes, exprimant un accord local entre différents partenaires, accord généralement sanctionné par la reconnaissance des institutions de niveau supérieur (l'État en particulier). On peut citer les chartes de parcs naturels régionaux ou les chartes de pays, mais aussi les chartes locales pour l'environnement, les chartes de territoire (sans statut officiel), les chartes paysagères, etc. Au statut juridique peu contraignant (sauf exception limitée), ces chartes expriment la constitution d'un collectif d'acteurs qui se reconnaissent dans un projet pour le territoire, instaurant une forme de gouvernance territoriale nouvelle (Moquay et al., 2000).

À partir de proximités géographiques (les participants étant caractérisés par l'ancrage dans un territoire), ces relations contractuelles tendent à établir les responsabilités respectives des acteurs et à organiser leurs relations de coopération et les conditions de leur intervention. Elles fournissent une modalité institutionnelle de construction ou de renforcement de proximités organisées, par l'établissement et la formalisation de relations stables entre acteurs. Le contrat est ici la traduction institutionnelle d'accords informels entre les acteurs (Poulle et Gorgeu, 1997). Ce faisant, ces politiques contractuelles territorialisées se trouvent à la 
confluence de diverses évolutions de l'action publique (Gaudin, 1991) : changements dans les modes d'intervention de l'État, transformation des modalités de la représentation politique, revalorisation des identités locales. D'autant plus qu'au réagencement des relations entre institutions (publiques et privées) qu'incarne ce mouvement de contractualisation, s'ajoute la recherche de nouvelles modalités de coopération entre institutions, ou d'implication directe des acteurs locaux dans l'action publique.

\section{Les coordinations résiliaires, entre local et régional}

Parmi les incitations publiques, les réseaux composent d'autres modalités de mise en place de la gouvernance locale. La politique des « réseaux de villes » en constitue une illustration, par l'élaboration du projet commun et par l'identification de limites dans la mise en place d'une gouvernance locale autour de proximités géographiques ou organisationnelles. Ces réseaux, au-delà des relations entre institutions municipales, sont fréquemment le support d'une formalisation de la participation des acteurs économiques et sociaux, voire de la « société civile » à la définition des actions (Eissel, 1999).

L'idée des réseaux de villes est lancée en 1991 sur un constat de faiblesse de l'armature urbaine française ${ }^{9}$, et de concurrence entre villes européennes. Sous l'impulsion du ministre chargé de l'aménagement du territoire $^{10}$ et relayée par la DATAR, elle encourage un rééquilibrage du territoire national s'appuyant sur le potentiel de développement des villes moyennes et de leur environnement proche. Elle envisage une autre armature urbaine ; de façon implicite la concentration urbaine est synonyme de

\section{Cf. Rapport Guichard 1986.}

10. Circulaire $n^{\circ} 3678 / \mathrm{SG}$ du 17 avril 1991 relative aux chartes d'objectifs et réseaux de villes, Bulletin officiel, Premier ministre, 91/2, p. 3-9. création de richesse et d'innovations qui structurent le territoire. C'est enfin une démarche reconnue au niveau européen, où un développement cohérent de l'espace communautaire s'appuie sur les réseaux de villes et un développement polycentrique (Commission européenne, 1999). Enfin, cette initiative est consacrée et reconnue par la Loi d'orientation pour l'aménagement et le développement du territoire (LOADT) du 4 février 1995 et inscrite aux contrats de plan Etat-région 2000-2006 dans le cadre des volets territoriaux. Aujourd'hui, 29 réseaux de villes, reconnus par la DATAR, sont en place.

La démarche d'identification des réseaux de villes repose sur un double mouvement : une initiative régionale qui émane des collectivités locales urbaines concernées, en termes de projet de développement économique et social, et de partenariat privé/public ; un cadre national et européen de rééquilibrage territorial dans une approche d'armature urbaine polycentrique. Le réseau de villes devient, par l'adoption de positions et d'actions communes, un acteur à part entière, répondant à une charte d'objectifs et trouvant son inscription dans le contrat de plan Etat-région.

Les premières constatations réalisées sur le fonctionnement de ces réseaux de villes mettent en évidence l'incontournable nécessité de la volonté politique, sans laquelle le réseau ne serait que le cadre de mise en œuvre d'une ou de plusieurs actions isolées (Hau-Rouchard et al., 1996). Les troisième et quatrième rencontres (1995 et 1996) du Club des réseaux de villes soulignent ainsi le nécessaire partenariat entre collectivités locales et acteurs socio-économiques alors même que le développement économique est à la base de la création de nombre de ces réseaux. De plus, en termes de projet, c'est bien l'extension de la notion de réseau à celle de territoire et la nécessaire élaboration d'un projet d'ensemble qui sera déterminante, et non la mise en œuvre d'actions juxtaposées tenant lieu de projet commun. 
L'exemple d'un autre type de réseau, celui de la Communauté de travail des villes alpines, éclaire la construction de la dimension collective. Ainsi, Fourny (2001), dans le cas des villes du Sillon alpin (Grenoble, Chambéry, Albertville, Annecy), souligne l'impact de l'auto-référence à l'identité alpine et territoriale sur le projet commun : "L'affirmation d'alpinité constitue également un acte fondateur de "confiance" [...] dans un ensemble de partenaires où l'interconnaissance est faible; [...] apparaît la nécessité d'une qualité reconnaissable par chacun », marquant ainsi, dans cette construction, le rôle de la proximité organisationnelle fondée sur l'appartenance. Ces logiques remettent en cause le système pyramidal et hiérarchique des rapports entre les villes et montrent la construction dans le temps (de 1992 à 1999) du projet commun et de l'action collective à mener (portée par la "Conférence" réunissant l'ensemble des maires des villes concernées). Il ne s'agit pas tant d'un réseau spatial que de la construction d'un réseau d'acteurs se référant aux mêmes valeurs, d'une forme de gouvernance où les règles sont élaborées en commun et les objectifs négociés. Cette construction permet de " transgresser un ordre territorial reposant sur un principe de continuité et de limites »(Fourny, op. cit.).

Ainsi, les réseaux de villes illustrent bien un nouveau niveau de gouvernance, fondé sur des partenariats transversaux aux hiérarchies et aux découpages fonctionnels (Offner et Pumain, 1996). Ce caractère novateur se retrouve dans le programme porté (collaborations internes, développement des solidarités, harmonisation des fonctions et des équipements structurants) et les moyens mis à disposition (prises de position communes, réalisation d'actions et d'opérations communes, participation aux procédures contractuelles). Programmes et moyens témoignent ainsi du projet collectif et de la volonté politique déterminants pour activer une proximité organisationnelle, voire géographique bien que non contiguë, condition de mise en œuvre de la gouvernance. Pour compléter, Unal (2000) souligne le nécessaire renforcement des relations citoyens/élus et le «porter de l'action collective à la connaissance $d u$ public $\gg$ dans la gouvernance locale. Cela rejoint la mise en réseau des acteurs pour lesquels Filippi et Torre (2003) mettent également l'accent sur l'utilisation des ressources de la proximité organisée, en parlant de mobilisation de compétences locales et de mise en valeur du territoire.

\section{La démocratie participative : du conseil de quartier à la gouvernance territoriale}

De nombreux dispositifs entendent traduire, dans les fonctionnements institutionnels, des préoccupations de proximité entre les décideurs et les citoyens. Un projet de loi récemment adopté " a ainsi repris l'expression « démocratie de proximité » pour regrouper divers dispositifs destinés à faciliter l'expression et la participation de la population dans les processus locaux de décision. Dans les années 1970, la question de la démocratie participative avait déjà émergé dans les débats politiques locaux, sous l'influence notamment des Groupes d'action municipale (GAM). Après un reflux dans les années 1980, la question de la participation émerge à nouveau dans les années 1990 à travers d'abord des politiques sociales (politique de la ville) puis par la mise en avant de l'élaboration, à chaque niveau, de projets collectifs de développement.

La rhétorique de la proximité est au cœur de ces démarches participatives. Elles postulent, en effet, que les programmes répondront d'autant mieux aux besoins réels des populations qu'ils auront été définis au plus près de leurs bénéficiaires, et autant que possible par eux. Ainsi, une procédure telle que le budget participatif entend réorienter les programmes publics vers les actions qui auront été jugées prioritaires par les habitants des quartiers concernés (Genro et De Souza,

11. Loi du 27 février 2002 sur la démocratie de proximité. 
1998). C'est donc d'abord au nom d'une proximité géographique que la démocratie participative est prônée. Mais elle renvoie in fine à des proximités organisationnelles, soit parce qu'elle s'appuie sur des structures sociales (associations, relations de quartier) préexistantes, soit parce qu'elle suscite la mise en place de tels dispositifs (conseils de quartier...).

La mise en œuvre de ces formes de participation suppose l'invention de modalités renouvelées de partage du pouvoir. La « société civile » ne participera durablement que si elle dispose d'un pouvoir réel dans les processus de décision, lui permettant de peser sur l'élaboration des choix politiques et la mise en œuvre des actions. L'enjeu principal d'une gouvernance se réclamant de la démocratie participative est donc l'acceptation d'une pluralité des formes de légitimité et l'articulation entre les lieux et les formes de pouvoirs ainsi établis (et éventuellement réformés), à différentes échelles.

Cette ambition démocratique, outre qu'elle heurte certaines conceptions traditionnelles de la politique et des institutions publiques, relevant notamment du « modèle républicain » en France, doit faire face à deux écueils :

- le premier est d'ordre méthodologique : la participation n'est pas chose spontanée, et suppose une organisation adaptée, des dispositifs ouverts et susceptibles de susciter l'expression des différents groupes concernés. Cela ne va pas de soi, et nombre de déclarations d'intention peinent à être traduites en actes (Gregory, 2000) ; - le second, qui découle du premier, est d'ordre sociologique, mais avec des implications politiques et éthiques. La propension à participer des différents groupes sociaux étant différenciée, comment s'assurer d'un équilibre dans l'expression des intérêts, de façon à ce que l'ouverture de nouvelles possibilités de participation ne renforce pas l'exclusion de certains dans les processus de décision (Godbout, 1983)?

\section{Conclusion \\ De l'intérêt porté au développement local, à la gouvernance locale et aux proximités}

Cet article a permis, par le jeu d'un croisement de perspectives entre sciences économique et politique, de situer la convergence de leurs approches dans la gouvernance locale, intégrant mécanismes productifs et mécanismes institutionnels dans les dimensions locale et local-global. Sur la base de trois modalités - procédures contractuelles, coordinations résiliaires et démocratie participative -, et au regard des fondements théoriques de la gouvernance locale examinés au préalable, un certain nombre de problèmes et de limites ont été rencontrés dans la mise en œuvre de démarches visant à mobiliser la proximité au profit d'objectifs de développement. Le décalage entre les principes des politiques se réclamant de la gouvernance et leur mise en œuvre pratique soulève, en effet, de nouvelles questions analytiques. Nous tenterons d'en explorer deux, qui renvoient aux faiblesses des politiques de proximité et recèlent quelques incitations à la modestie. La première porte sur la nature de la relation entre proximités et gouvernance locale; la seconde a trait au décalage entre les principes des politiques qui se réclament de la gouvernance et leur mise en œuvre pratique.

L'analyse des fondements théoriques, économiques et politiques, du concept de gouvernance met en évidence une relation proximité-gouvernance qui ne tient pas tant à un rapport de « cause à effet » mais de convergences d'interprétations. Ainsi dans le cas des approches économiques, la recherche de théorisation des dynamiques territoriales par la convergence de différents types de proximités fait émerger le concept de gouvernance dans l'interprétation du fait productif. Il y a donc une dimension explicative de la gouvernance par les proximités, bien que la proximité 
géographique soit parfois remise en cause, comme dans les réseaux de villes où la contiguiité spatiale au sens strict disparaît. En revanche, l'approche des sciences politiques centrée sur l'analyse des modes territoriaux de gouvernance ne traite qu'implicitement les dimensions de la proximité, mais révèle néanmoins une convergence d'intérêt pour ces deux objets que sont la gouvernance et les proximités. Finalement, l'existence de formes de proximité est une condition de la gouvernance locale, mais ne saurait en être la cause suffisante. En particulier, la gouvernance ne saurait se passer d'un projet commun, où les seules proximités ne peuvent être à l'origine de l'initiative.

D'un point de vue pragmatique, un paradoxe est à l'origine des limites manifestes rencontrées par les politiques publiques qui se réclament de la gouvernance, et qui tentent d'impulser artificiellement des dynamiques de développement territorial. Sur la base d'expériences locales réussies, de success-stories, on entend mettre en œuvre des modalités génériques ou systématiques de renforcement volontariste des proximités entre acteurs. On passe de la singularité d'itinéraires de développement propres à un territoire et à des configurations particulières d'acteurs et d'activités à des modalités normalisées et universelles d'organisation des acteurs. Il y a bien là une véritable dénaturation des démarches ascendantes, qui voudrait systématiser des expériences locales et universaliser le singulier.

On en trouvera quelques exemples édifiants dans les dispositifs récents de développement territorial. Ainsi, de la mise en place expérimentale de 42 pays de préfiguration, on passerait sans hésitation à la perspective d'une couverture uniforme du territoire national par 300 à 400 pays. Ou encore, sur la base de l'analyse de situations spécifiques effectivement florissantes (les districts industriels de la troisième Italie), on entend fabriquer du système productif local à la demande... Enfin, le légis- lateur n'a pas hésité (par l'obligation de créer des conseils de quartier dans toutes les communes de plus de 80000 habi$\operatorname{tants}^{12}$ ) à imposer à la fois une obligation de pratique et une forme préétablie à la participation des citoyens au débat local, véritable injonction contradictoire qui fait l'impasse sur la diversité des pratiques, des formes de mobilisation, des enjeux locaux ${ }^{13}$.

La labilité du social, que les analyses en terme de proximité essaient de réintroduire dans la compréhension des phénomènes économiques, se trouve ainsi confrontée à des mécanismes de rigidité normative, relevant d'une logique de réduction politico-administrative. La confrontation de la diversité territoriale avec des politiques dont la logique est homogénéisante peut-elle réellement être productrice de développement ? À cette contradiction, qui voudrait imposer des formes d'organisation dont la richesse provient largement du caractère spontané, injonction descendante à la créativité ascendante, s'ajoute une autre dimension problématique, autour de la dimension temporelle des phénomènes. Les politiques d'encouragement à l'activation des liens de proximité négligent largement la dimension temporelle de construction de ces proximités. Cette construction est pourtant issue le plus souvent de processus d'apprentissages progressifs, d'interactions répétées qui prennent sens sur le long terme.

Pour toutes ces raisons, il apparaît que les ambitions placées dans les politiques publiques de développement tablant sur l'activation de la proximité relèvent pour une large part d'une illusion. Contrairement aux

12. Loi du 27 février 2002 sur la démocratie de proximité.

13. La loi Voynet du 25 juin 1999 avait en revanche évité de tomber dans ce piège de la formalisation et de l'uniformisation en ne fixant qu'un cadre très lâche (trop au regard de certains observateurs) à l'organisation des conseils de développement des pays. 
institutions formelles, la gouvernance locale se construit, mais ne se décrète pas; de même, les proximités peuvent - voire doivent - être activées, mais cette activation obéit aux temps et aux formes d'établissement des relations entre acteurs, et est donc rétive aux injonctions. Si la proximité est bien une opportunité, voire une condition du développement, elle n'en constitue pas la clé systématique

Les auteurs tiennent à remercier les relecteurs pour leurs commentaires et suggestions d'amélioration sur une première version de cet article.

\section{RÉFÉRENCES BIBLIOGRAPHIQUES}

Aydalot $\mathrm{Ph}$. À la recherche des nouveaux dynamismes spatiaux. In «Crise et Espace », Economica, Paris, 1984, p. 37 59.

Bagnasco A., Trigilia C. La construction sociale du marché, le défi de la troisième Italie. Les éditions de l'École Normale Supérieure de Cachan, Collection Sciences Sociales, 1993, 284 p.

Becattini G. Les districts industriels en Italie. In « La flexibilité en Italie », TERRMIRE, 1989, p. 261-270.

Benko G., Lipietz A. Des réseaux de districts aux districts de réseaux. In Benko G., Lipietz A. " Les régions qui gagnent. Districts et réseaux : les nouveaux paradigmes de la géographie économique », PUF, Paris, 1992, p. 379-388.

Berthet T., Palard J. Culture politique réfractaire et décollage économique. L'exemple de la Vendée du Nord-Est. Revue française de science politique, 1997, vol.47, $n^{\circ} 1$, p. 29-47.

Beslay C., Guillaume R., Grossetti M., Taulelle F., Salles D., Daynac M. La construction des politiques locales: reconversions industrielles et systèmes locaux d'action publique. L'Harmattan, Paris, 1998, $220 \mathrm{p}$.

Cannac Y., Godet M. La “ bonne gouvernance ". L'expérience des entreprises, son utilité pour la sphère publique. Futuribles, 2001, n 265, p. 41-50.

Castells M., Godard F. Monopolville, l'État, l'urbain. Mouton, Paris, 1974.
Coase R. H. The Nature of the Firm. Texte original, 1937, traduction française : $L a$ nature de la firme, Revue Française d'Economie, 1987, p. 133-163.

Commission européenne. Schéma de développement de l'espace communautaire (SDEC). CE, Potsdam, 1999, 89 p.

Commons J. R. Institutional Economics. University of Wisconsin Press, Madison (WIS), 1934.

Corolleur F., Pecqueur B. Les politiques économiques locales en France durant la décennie 1980. La construction de spécificités territoriales comme enjeu stratégique pour les collectivités locales. In Demazière C., (Ed.) « Du local au global, les initiatives locales pour le développement économique en Europe et en Amérique », L'Harmattan, Paris, 1996.

Courlet $\mathrm{Cl}$., Pecqueur B. Les systèmes industriels localisés en France : un nouveau modèle de développement. IREP-D, Grenoble, 1989.

Dente B. Metropolitan Governance Reconsidered or how to Avoid Errors of the Third Type. Governance, 1990, volume 3, $\mathrm{n}^{\circ} 1$. Dockès P. L'espace dans la pensée économique du XVr au XVIIr siècle. Flammarion, Nouvelle bibliothèque scientifique, Paris, 1969, $461 \mathrm{p}$.

Eissel D. Kommunale Netzwerke als neue Formen konzertierter Aktion. In Klotz J., Zielinski H., Europa 2000. Lokale Demokratie im Europa der Regionen, Heilbronn, Distel Verlag, 1999, p. 171-190. 
Filippi M., Torre A. L'organisation en réseau : de l'instauration de règles locales aux actions collectives. In Dupuy C. et Burmeister A., (Eds) « Entreprises et territoires : les nouveaux enjeux de la proximité », La Documentation française, Paris, 2003.

Fourny M.-Ch. Le réseau des villes-centres de Rhône-Alpes, acteur d'une nouvelle construction du territoire régional. In Jouve B., Spenlehauer V., Warin Ph., (Dir.) « La région laboratoire politique: une radioscopie de Rhône-Alpes ", La Découverte "recherche", Paris, 2001, p 245-262.

Gaudin J.-P. (Dir.). La négociation des politiques contractuelles. L'Harmattan, Paris, 1996.

Gaudin J.-P. Du jacobinisme au modèle américain? Faire la politique : le chantier français. Autrement, 1991, n 122 , p. 128-138.

Gaudin J.-P., Novarina G. (Dir.). Politiques publiques et négociation : multipolarités, flexibilités. Editions du CNRS, Paris, 1997, $174 \mathrm{p}$.

Genro T., De Souza U. Quand les habitants gèrent vraiment leur ville. Le budget participatif : l'expérience de Porto Alegre au Brésil. Editions Charles Léopold Mayer, Paris, 1998, 110 p.

Gilly J.-P., Pecqueur B. Régulation et territoire, une approche des dynamiques institutionnelles locales. Communication au colloque « Proximité et Coordination économique », Lyon, 5 et 6 mai 1997, 19 p.

Gilly J-P., Leroux I. Vers une approche institutionnaliste de la dynamique des territoires. Revue d'Économie Régionale et Urbaine, 1999, n 1, p 93-114.

Gilly J-P., Torre A. Dynamiques de proximité. L'Harmattan, Paris, 2000, 310 p. Gilly J-P., Wallet F. Forms of Proximity, Local Governance and the Dynamics of Local Economic Spaces: the Case of Industrial Conversion Processes. International Journal of Urban and Regional Research, 2001 , vol. 25.3 , p. 553-570.
Godbout J. La participation contre la démocratie. Éditions coopératives Albert StMartin, Montréal, 1983, 187 p.

Gregory A. Problematizing Participation. Evaluation, 2000, n², pp. 179-199.

Grossetti M., Beslay Ch. La construction des politiques locales de reconversion industrielle. Revue d'Économie Régionale et Urbaine, 1999, n' 1 , p.63-92.

Guichard O. (Rapport). Propositions pour l'aménagement du territoire. Rapport au ministre de l'Equipement, du Logement, de l'Aménagement du territoire et des Transports ; commission de réflexion sur l'aménagement du territoire, La Documentation française, Paris, 1986.

Gurr T. R., King D. S. The State and the City. Macmillan, New York, 1987, 218 p.

Hau-Rouchard M-F., Royaux D., Tesson F., Thoviste L. La politique des réseaux de villes de la DATAR. In Données urbaines 1 , chapitre $\mathrm{V}$ « Des villes en système $", 1996$, p. 333-340.

Jayet H. Organisation et concurrence territoriale : un cadre global d'analyse, Communication au colloque IFRESIPIRVilles, Lille, 1-18 mars 1994.

Jouve B. La gouvernance d'agglomération en Europe : les leçons d'un échec. Pouvoirs locaux, 1999, n 42, p. 62-69.

Krugman P. Increasing Returns and Economic Geography. Journal of political Economy, 1991, 99(3), p. 483-499.

Lagroye J. Société et politique. Jacques Chaban-Delmas à Bordeaux. Pédone, Paris, 1971.

Le Galès P. Du gouvernement des villes à la gouvernance urbaine. Revue française de science politique, 1995, volume 45, $n^{\circ} 1$, p. 57-95.

Lefèvre C. Gouvernements métropolitains et gouvernance dans les pays occidentaux. Politiques et management public, 1998, volume $16, n^{\circ} 2$, p. $35-60$.

Logan J.R., Molotch H.L. Urban Fortunes. The Political Economy of Place. University of California Press, Berkeley, 1987. 
Lorrain D. Administrer, gouverner, réguler. Annales de la recherche urbaine, 1998, $n^{\circ}$ 80-81, p. 85-92.

Lorrain D. De l'administration républicaine au gouvernement urbain. Sociologie du travail, $1991, \mathrm{n}^{\circ} 4$.

Lorrain D. La montée en puissance des villes. Economie et humanisme, 1989, $\mathrm{n}^{\circ} 305$.

Mabileau A. Le système local en France. Montchrestien, coll. Clefs, Paris, 1994.

Marshall A. Principle of Economics. MacMillan, Londres, 1920.

Moquay P., Gorgeu Y., Poulle F., Balligand J.P. L'accord local - contrat moral. Aux fondements des territoires. Actes et commentaire du séminaire «Gouvernance des territoires de chartes $"$, Mairieconseils/Éditions du développement territorial, 2000, $137 \mathrm{p}$.

Moquay P., Palard J. Représentation socioprofessionnelle et espace régional. Le Comité économique et social d'Aquitaine (1982-1989). Talence, CERVL-IEP de Bordeaux, 1990, $110 \mathrm{p}$.

Moquay P. Sentiments d'appartenance et développement régional. In Côté $\mathrm{S}$. et Proulx M.-U. (dir.), « Espaces en mutation ", Rimouski (Qc), GRIDEQ-GRIR, coll. Tendances et débats en développement régional, 1998, p. 57-69.

Offner J.-M. Gouvernance, mode d'emploi. Pouvoirs locaux, 1999, n42, p. 58-61.

Offner J-M., Pumain D. (Dir.). Réseaux et territoires. Significations croisées. Ed de l'Aube, La Tour d'Aigues, 1996.

Padioleau J.-G. L'action publique moderniste après la décentralisation. Politiques et management public, 1991, volume 9 , $n^{\circ} 4$.

Padioleau J.-G. L'action publique postmoderne : le gouvernement politique des risques. Politiques et management public, 1999 , volume $17, n^{\circ} 4$, p. 85-127.

Padioleau J.-G. La gouvernance ou comment s'en débarrasser. (Stratégies de corruption). Espaces et sociétés, 2000, $\mathrm{n}^{\circ}$ 101-102, p. 61-73.
Parks R. B., Oakerson R. J. Metropolitan Organization and Governance. À local Public Economy Approach. Urban Affairs Quarterly, 1989, volume 25, n 1 , p. 1829.

Perroux F. Note sur les pôles de croissance. Economie Appliquée, 1955, 1-2, p. 307320.

Peterson P. City Limits. University of Chicago Press, Chicago, 1981, 268 p.

Piore M., Sabel C. The Second Industrial Divide. Basic Books, New York, 1984.

Polsby N. Community Power and Political Theory. Yale University Press, New Haven, 1980.

Poulle F., Gorgeu Y. Essai sur l'urbanité rurale : cinq territoires ruraux, leurs serments et leurs modes de gouvernement. Syros, coll. Les cahiers de l'intercommunalité, Paris, 1997.

Rallet A. De la globalisation à la proximité géographique : pour un programme de recherches. In Gilly J-P et Torre A. (Dir.) «Dynamiques de proximité », L'Harmattan, Paris, 2000, p. 37-57.

Rallet A., Torre A. Économie industrielle et économie spatiale : un état des lieux. In Économie industrielle et économie spatiale, Economica, Paris, 1995, p.3-37.

RERU (Revue d'économie régionale et urbaine). « Economie de proximité », 1993, numéro spécial $n^{\circ} 3$.

Ruegg J., Decoutere S., Mettan N. (Dir.). Le Partenariat public-privé : un atout pour l'aménagement du territoire et la protection de l'environnement?, Presses polytechniques et universitaires romandes, Lausanne, 1994.

Skocpol T. Bringing the State Back In: Strategies of Analysis in Current Research. In Evans P. B., Rueschemeyer D., Skocpol T. (Dir.), "Bringing the State Back In", Cambridge University Press, 1985, p. 3-37.

Soulage B. La place du politique dans les systèmes productifs localises. In Courlet C., Soulage S. (ed.), « Industrie, territoire et politiques publiques ", L'Harmattan, Paris, 1994. 
Stone C. N. Regime politics. Governing Atlanta, 1946-1988. University Press of Kansas, Lawrence, 1989.

Stone C. N. Urban Regimes and the Capacity to Govern. À Political Economy Approach. Journal of Urban Affairs, 1993, volume 15, $\mathrm{n}^{\circ} 1$, p. 1-28.

Thisse J.-F. Science régionale et économie géographique : matériaux pour un rapprochement. Revue d'économie régionale et urbaine, 1996, $n^{\circ} 4$, p. 673-694.

Unal M. Les mots-clés d'une circulaire : contribution sémantique et méthodologique à la maturation des intercommunalités au regard des perspectives données par le rapport Mauroy. Communication au colloque « Réseaux de Ville ", Paris, 7 décembre 2000.

Villeval M.-C. Une théorie économique des institutions? In Boyer R., Saillard Y. (dir.), "Théorie de la régulation, l'état des savoirs », La Découverte, Paris, 1995.
Wallet F. Gouvernance locale et action publique locale : l'exemple des processus de reconversion industrielle. Communication au colloque de l'ASRDLF, Lille, 1-3 septembre 1997, 33 p.

Weber A. Theory of the Location of Industries. The University of Chicago Press, 1929, réédité en 1957.

Williamson O. E. Markets and Hierarchies, Analysis and Anti-trust Implications. The Free Press, New York, 1975.

Williamson O.E. The Modern Corporation : Origins, Evolution, Attributes. Journal of Economic Literature, December 1981, volume XIX, p. 1 537-1 568.

Wolman H., Goldsmith M. Local Autonomy as a Meaningful Concept. Comparing Local Government in the United States and the United Kingdom. Urban Affairs Quarterly, 1990, volume 26, $\mathrm{n}^{\circ}$ 1, p. 3-27. 